\title{
Neutrino Factory R\&D and the International Design Study for the Neutrino Factory
}

\author{
Alain Blondel ${ }^{1}$ \\ DPNC, University of Geneva \\ Quai Ansermet 24, CH 1205 Geneva 4 \\ E-mail: Alain.Blondelecern.ch
}

\section{For the IDS-NF collaboration ${ }^{2}$}

The Neutrino Factory is the most powerful machine devised to study neutrino oscillations. It is also the first step towards muon colliders. A world-wide program of R\&D experiments and a design study are taking place at the moment, with the aim of delivering a credible design and cost estimate by 2012. The scientific highlights of this program are presented.

European Physical Society Europhysics Conference on High Energy Physics EPS-HEP 2009, Krakow, Poland, July 16-22, 2009 
Muon Collider and Neutrino Factory are novel accelerators and their development is of great interest for the future of the field of particle physics. R\&D for the Neutrino Factory is largely synergetic with that of the muon collider. The design of the Neutrino Factory has been developed since 1998[1] and has been the object of several physics or accelerator studies, and design studies, both in the USA[2] and in Europe [3]. A performance of $10^{21} 20 \mathrm{GeV}$ muon decays per year starting from a $4 \mathrm{MW}$ proton beam on target is now considered achievable. Obtaining a beam of $10^{14}$ muons/s is unprecedented, the most intense muon beam intensities at PSI are $10^{9}$ muons/s.

A program of experimental $R \& D$ has been launched to establish the feasibility of the most critical elements of the Neutrino Factory: the target experiment MERIT at CERN[4], the MUon COOLing R\&D program MUCOOL[5] at Fermilab, the Muon Ionization Cooling Experiment MICE[6] at RAL and the EMMA non-scaling FFAG electron demonstration at Daresbury Lab[7]. The International Scoping Study[8], initiated at the request of the RAL director in 2005, also covered to some extent the conventional neutrino superbeam and the beta-beam; it spelled out well-defined baseline parameters for the accelerator and detectors, to be brought forward for a more detailed study including cost estimate, the International Design Study (IDS-NF), which has been ongoing since 2007 [9]. The Plans of the IDS is to deliver an intermediate design report (IDR) in 2010 and a reference design report (RDR) in 2012.

The Neutrino Factory baseline layout is shown in Figure 1. A high power 4MW proton accelerator delivers a train of a few (3-5) short pulses ( $<3 \mathrm{~ns})$ on a high $\mathrm{Z}$ target embedded in a $20 \mathrm{~T}$ solenoid. The energy of the primary protons is not a very sensitive parameter, pion production is within $20 \%$ of the optimum in the energy range of 4-15 $\mathrm{GeV}$. The time structure is critical; a high power LINAC such as the CERN SPL has been shown to be an adequate possibility but requires two additional proton storage rings to elaborate the desired pulse trains. The feasibility of the target system was the aim of the MERIT experiment. The MERIT experiment has demonstrated the safe operation of a mercury jet in a $15 \mathrm{~T}$ solenoid; it measured the properties of the jet when exposed to instantaneous intensities similar to those of a $8 \mathrm{MW}$ proton beam; the effect of the time structure was investigated. What MERIT has not demonstrated is the feasibility and safety of a production target station operating at this high power. 


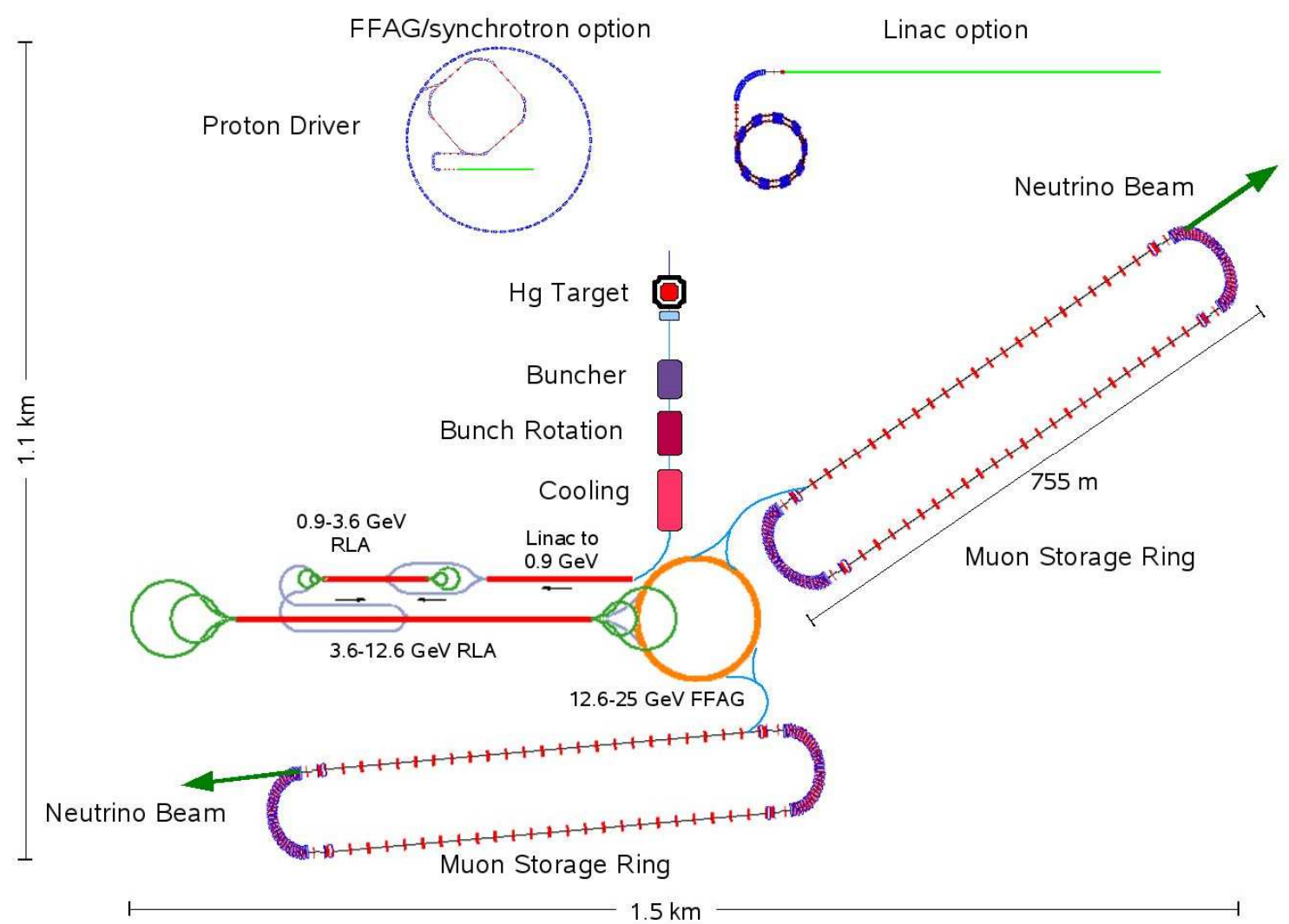

Figure 1 Layout to scale of the Neutrino Factory baseline

The muons of both signs produced from the decay of pions are spread over a large phase space. The reduction of longitudinal momentum is obtained by phase rotation and the transverse emittance reduction is performed by ionization cooling. Ionization cooling is the only possible technique given the short muon lifetime. It is a critical component in the performance and cost optimization of the Neutrino Facility, and even more for the Muon Collider. It has never been applied to muons in this energy range. It involves a succession of energy loss in low $\mathrm{Z}$ absorbers (Liquid Hydrogen or Lithium Hydride) and re-acceleration with RF cavities. The demonstration of the feasibility of a channel that performs according to calculations is the aim of the MICE experiment at RAL. MICE is presently under construction, having achieved first beams in 2008.

Throughout the first section of the accelerator, the muons of 100-300 MeV/c are contained in a longitudinal magnetic field in which the high gradient RF cavities are operated. Obtaining high accelerating gradient in magnetic field is the key accelerator physics challenge in cooling. This question is addressed specifically in the MUCOOL test program at FNAL, but will be applied in MICE.

Acceleration of the resulting beam must be extremely fast and perfectly synchronized. Synchrotron radiation by muons is suppressed and circular accelerators can be used, but the magnets must have fixed field. This leads to the use of a chain of recirculating linacs or fixed field accelerators with large momentum aperture (FFAG, Fixed Field Alternating Gradient), which can each typically accelerate the particles by a factor 2 . 
The principles of FFAG have been known since more than 50 years; scaling FFAGs have been constructed in the last 10 years for proton accelerators. For the neutrino factory a new version called non-scaling FFAG has been proposed for cost-saving purpose, making use of the fact that the muons remain only 10-20 turns in the accelerator. EMMA at Daresbury Lab aims at developing such a machine using electrons of similar velocity than the muons in the Neutrino Factory.

The accelerator can be designed in such a way as to accelerate both signs of muons simultaneously, as long as the neutrinos from $\mu^{+} \rightarrow e^{+} v_{e} \bar{v}_{\mu}$ are separated in time from those of $\mu^{-} \rightarrow e^{-} \bar{\nu}_{e} \nu_{\mu}$ in the detectors. The stored muon energy is defined to be 25 $\mathrm{GeV}$ in reference to the effective tau production threshold (around $15 \mathrm{GeV}$ ), and the matter resonance around $12 \mathrm{GeV}$. It is also well suited for maximal performance in detecting CP violation. The storage ring design is guided by the requirement of low angular divergence but is otherwise straightforward. There are no major issues of radiation or intensity in the storage ring.

Muon decays $\mu^{+} \rightarrow e^{+} \nu_{e} \bar{\nu}_{\mu}$ (and $\mu^{-} \rightarrow e^{-} \bar{\nu}_{e} \nu_{\mu}$ ) produce simultaneously two types of neutrinos. The appearance signal of most interest is $\left\{v_{e} \leftrightarrow v_{\mu}\right.$; $\left.v_{\mu}+N \rightarrow \mu^{-}+X\right\}$ (wrong sign muons) which must be separated from the nonoscillated signal $\bar{v}_{\mu}+N \rightarrow \mu^{+}+X$ (right sign muons) by magnetizing the detector. This is a severe constraint and prevents from using the large liquid detectors foreseen for the search for proton decay. Wrong sign muon detection can be performed with very little background with a magnetized iron neutrino detector (MIND) readout with scintillator. Based on the cost and performance of MINOS, NOvA and of the T2K near detector system, which demonstrate the progress made in light detection with avalanche photodiodes, a detector with a fiducial volume of $100 \mathrm{kton}$ is considered. The MIND is also capable of measuring the muon neutrino disappearance and the neutral current channels. Other detector types are required for tau detection or for the detection of the wrong sign electrons; magnetized versions of the Totally Active Scintillator Detector (TASD), Liquid Argon or Emulsion detectors have been described in the ISS detector study. One of the main challenges is the design of an open magnet of this size.

Near detector systems are presently under simulation and design. By combination of muon flux measurement and measurement of the energy by muon spin precession the neutrino fluxes can be predicted with high accuracy. The high flux at the near detector allows significant samples of purely leptonic reactions to be observed, thus providing a normalization against which precise measurements of hadronic cross-sections for $v_{\mu}, v_{e}, \bar{v}_{\mu}, \bar{v}_{e}$ become possible.

The precision that can be reached by experiments at a neutrino factory is typically one or more order of magnitude better for the oscillation parameters $\theta_{23} \theta_{13} \Delta \mathrm{m}_{13}^{2}$ and for the $\mathrm{CP}$ violating phase $\delta$ than for any other machine considered so far, with the possible exception of high gamma beta-beams. The sensitivity reach for measuring the parameter 
$\theta_{13}$, should it be very small, is also better than for any other facility considered. Unlike most set-up sondidered, the Neutrino Factory with two long baselines and a full analysis of the energy spectrum of appearance events is able to solve degeneracies and ambiguities completely over most of the parameter space that it is sensitive to. The ability to measure interactions of tau neutrinos offers unique possibilities to test the unitarity of the neutrino mixing matrix at either far or near detector stations. The optimum setup for a neutrino factory is mildly dependent on the existing knowledge on the value of the mixing angle $\theta_{13}$. In particular one can note, as an existence proof, that there is a pair of baselines that are close enough to the optimum baselines stated by the ISS: CERN to Pyhasalmi in Finland $(2285 \mathrm{~km})$, which is also a LAGUNA site, and CERN to INO in India $(7152 \mathrm{~km})$.

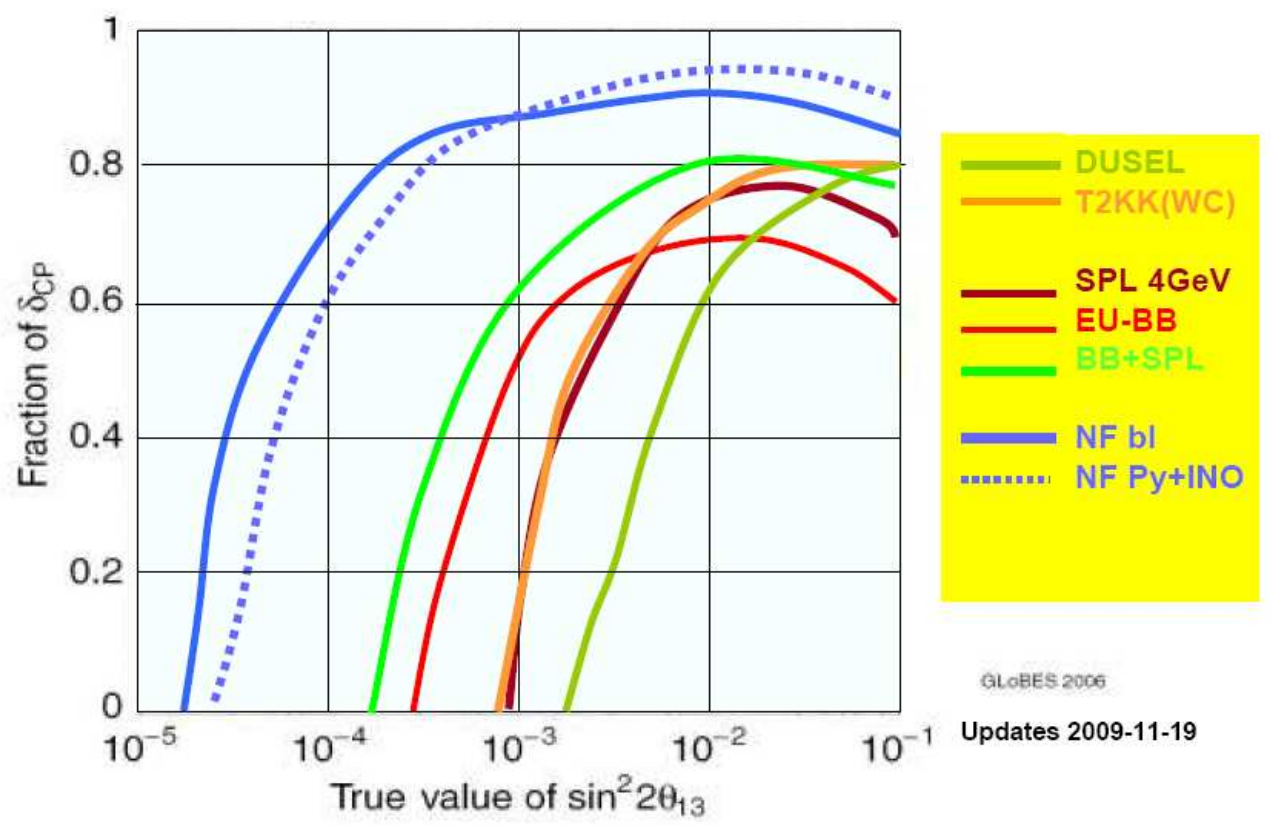

Figure 2 Compilation of $3 \sigma \mathrm{CP}$ sensitivities of future long baseline projects. Here the fraction of $\delta_{\mathrm{CP}}$ where CP violation can be observed at 3 standard deviations is plotted as a function of $\theta_{13}$. T2KK: T2K 1.66 MW beam to $270 \mathrm{kton}$ fid volume Water Cherenkovs detectors in Japan (295km) and in Korea $(1050 \mathrm{~km})[10]$; DUSEL: a WBB from Fermilab to a $300 \mathrm{kton}$ WC in Dusel (1300km)[11]; SPL $4 \mathrm{GeV}$, EU-BB and BB+SPL curves stand for the CERN to Fréjus (130km) project [12]; NF baseline is the Neutrino Factory baseline $(4000 \mathrm{~km}$ and $7000 \mathrm{~km}$ baselines) and NF Py+INO represents the concrete baseline from CERN to Pyhaslami mine in Finland (2285 km) and to INO in India $(7152 \mathrm{~km})[13]$.

The decision of if, when and where to build a neutrino factory will be determined by several factors, including, at any given time, the perceived relative feasibility and reach of more conventional alternatives. The CERN strategy document recommends the information to be available in 2012. This is the goal pursued by all participants in the world wide R\&D program. 


\section{References}

[1] S. Geer, Neutrino Beams From Muon Storage Rings: Characteristics And Physics Potential, Phys.Rev.D57:6989-6997,1998, Erratum-ibid.D59:039903,1999]

[2] N. Holtkamp and D. Finley. eds., "A Feasibility Study of a Neutrino Source Based on a Muon Storage Ring," Fermilab-Pub-00/108-E, 2000;

S. Ozaki, R. Palmer, M. Zisman, and J. Gallardo, eds., "Feasibility Study-II of a Muon-Based Neutrino Source," BNL-52623 (2001); see http://www.cap.bnl.gov/mumu/studyii/final_draft/The-Report.pdf;

S. Geer and M. Zisman, eds., "Neutrino Factory and Beta Beam Experiments and Development," http://www.cap.bnl.gov/mumu/study2a/.

[3] B. Autin, A. Blondel, J. Ellis eds., "Prospective Study of Muon Storage Rings at CERN", CERN99-02, ECFA/99/197 (April 1999);

A. Blondel et al. eds., ECFA / CERN Studies of a European Neutrino Factory Complex, CERN2004-002, ECFA/04/230, (April 2004).

[4] K.T.McDonald et al., "The MERIT high-power target experiment at the CERN PS," PAC09 Vancouver, http://www.hep.princeton.edu/ mcdonald/mumu/target/pac09/tu4gri03.pdf

[5] http://mice.iit.edu/mta/

[6] MICE Status Report -- October 2009 MICE Collaboration, Y. Karadzhov et al, http://mice.iit.edu/micenotes/public/pdf/MICE0267/MICE0267.pdf

[7] EMMA - the world's first non-scaling FFAG, R. Edgecock et al, Proceedings of EPAC08, Genoa, Italy, THPP004. http://accelconf.web.cern.ch/AccelConf/p01/PAPERS/TPAH137.PDF

[8] The ISS Accelerator Working group (M. Apollonio et al.), “Accelerator design concept for future neutrino facilities", 2009 JINST 4 P07001

The ISS Detector Working group (T. Abe et al.) "Detectors and flux instrumentation for future neutrino facilities", 2009 JINST 4 T05001

The ISS Physics Working group (Bandyopadhyay et al.) "Physics at a future Neutrino Factory and super-beam facility”, Rep. Prog. Phys. 72 No 10 (Oct. 2009) 106201

[9] https://www.ids-nf.org/

[10] T2KK: F. Dufour, T. Kajita, E. Kearns, and K. Okumura: Further study of neutrino oscillation with two detectors in Kamioka and Korea, NP08, KEK, 2008; a 100 kton of LArg TPC at the far location would give similar sensitivity.

[11] DUSEL, V. Barger et al Fermilab-081-AD-E Report on the long baseline neutrino experiment study (2006); a 50kton LArg in DUSEL would give similar sensitivity.

[12] Jean-Eric Campagne, Michele Maltoni, Mauro Mezzetto and Thomas Schwetz, Physics potential of the CERN-MEMPHYS neutrino oscillation project, JHEP04(2007)003. Curves recalculated by T. Schetz-Mangold to take into account the present baseline intensities for the beta beam.

[13] W. Winter, private communication, A. Blondel, 4thIDS-NF meeting in Mumbai, https://www.ids-nf.org/wiki/TIFR-2009-10-12/Agenda/Files?action=AttachFile \&do=get\&target=S4-Blondel-1-v2.pdf 